\title{
Optimal Materials Selection for Bimaterial Piezoelectric Microactuators
}

\author{
Prasanna Srinivasan and S. Mark Spearing, Member, ASME
}

\begin{abstract}
Piezoelectric actuation is one of the commonly employed actuation schemes in microsystems. This paper focuses on identifying and ranking promising active material/substrate combinations for bimaterial piezoelectric (BPE) microactuators based on their performance. The mechanics of BPE structures following simple beam theory assumptions available in the literature are applied to evolve critical performance metrics which govern the materials selection process. Contours of equal performance are plotted in the domain of the governing piezoelectric material properties ( $d$ coefficients, elastic modulus, coupling factors and dielectric constants) for commonly employed substrates to identify optimal material combinations for various functional requirements. The influence of materials selection on the actuation efficiency, quality factor and the electromechanical impedance is also discussed. Selection of a suitable actuation mechanism for a boundary layer flow control application is illustrated by comparing the performance limits of BPE and bimaterial electrothermal actuators considering the constraints on the functional requirements imposed by the associated microfabrication routes.

[2007-0047]
\end{abstract}

Index Terms-Electromechanical effects, electrothermal effects, microactuators, piezoelectric materials, scaling and materials selection.

\section{INTRODUCTION}

$\mathbf{M}$ ATERIALS selection is a critical process in the design cycle of all engineering systems. The design of microelectromechanical systems (MEMS) in particular is challenging because of the availability of a limited range of materials [1] to meet increasingly multifunctional requirements. Although the selection of suitable actuation mechanisms has been illustrated using performance maps at macro and microscales [2], [3], the potential of MEMS technology can be fully realized only if a wider range of promising candidate materials is explored. Therefore, the design guidelines for selecting suitable materials for various functional requirements need to be established for further development. This paper focuses on identifying optimal material combinations for bimaterial piezoelectric (BPE) microactuators.

Piezoelectric microactuators in general, are capable of delivering relatively large forces $(10 \mu \mathrm{N}-1 \mathrm{mN})$ but are limited to small displacements $(0.1-10 \mu \mathrm{m})$. Present generation mi-

Manuscript received February 26, 2007; revised October 26, 2007. The work of P. Srinivasan was supported by the University of Southampton, the Overseas Research Students Award Scheme and a Royal Society-Wolfson Research Merit Award. Subject Editor D. DeVoe.

The authors are with the School of Engineering Sciences, University of Southampton, Southampton, SO17 1BJ, U.K. (e-mail: prasanna@ soton.ac.uk; spearing@soton.ac.uk).

Digital Object Identifier 10.1109/JMEMS.2008.918398 crosystems have generally preferred ferroelectric piezoceramics such as $\mathrm{BaTiO}_{3}$, PZT, PZN-PT, PMN-PT, and PYN-PT due to their high $d$ coefficients and reasonably high Curie temperatures, which are compatible with most transducer applications. Despite their promising characteristics, applications of piezoelectrics in commercial microsystems are not widely prevalent but do include the important case of inkjet printer heads [4] and noncontact atomic force microscope probes [5]. However, a much wider range of research or prototype applications of piezoelectrics in MEMS include linear micromotors [6]-[8], high power transformers [9], micropumps [10], [11], and power generators [12].

The optimal selection of a suitable active material for a given elastic substrate is of great importance from the MEMS perspective considering the need to develop reliable devices with high performance. This challenge is equally supported by the growing interest in developing thin films of arbitrary materials on various substrates thereby improving the capability of existing microfabrication routes. Therefore, guidelines for optimal materials selection in the preliminary design stage become essential. These have not yet been established for microsystems despite the hitherto limited available choices of the active materials [13]-[15]. This paper is aligned with the aims of previous studies on identifying promising candidate materials for bimaterial electrothermal (BET) actuators to meet defined functional requirements [16], [17], properly accounting for the discrete nature of the properties of engineering materials. The key objectives of this paper are to identify and rank optimal combinations of active materials and elastic substrates to deliver maximum performance against the functional requirements and to compare the performance of electrothermal and piezoelectric actuators in bimaterial architectures at microscales. The performance metrics considered are tip slope (displacement), blocked moment (force), work/volume and frequency. Tip slope is a critical performance metric for applications such as micromirror devices which requires large out of plane deflection. Blocked moment (force) is a governing metric for microflow control devices such as fluid-mixers in biochips. Work per volume is an important metric for applications such as boundary layer flow control actuators and fiber-optic switches. The influence of materials selection on the quality factor $(Q)$ and the electromechanical impedance of actuators are also discussed in this paper.

This paper assumes linear elastic material behavior for the actuator design in the estimation of the performance. This assumption restricts the impact of the results; however, in practice a majority of piezodevices operate within the linear range. 


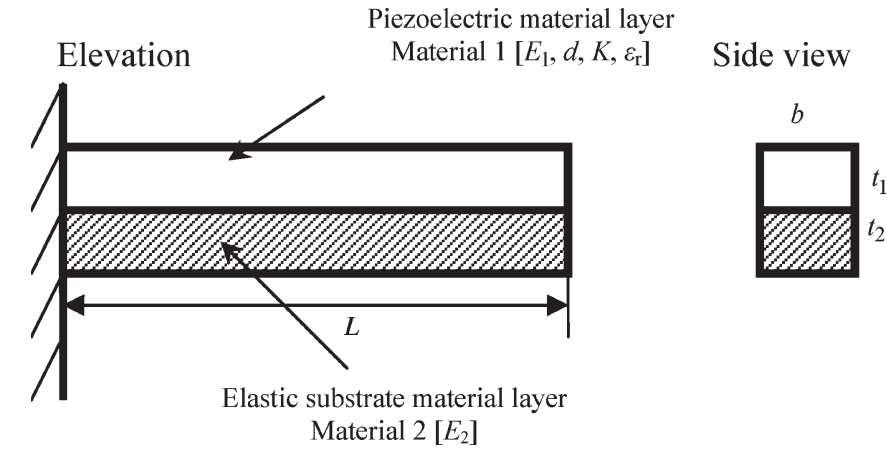

Fig. 1. Schematic of a cantilever BPE actuator structure.

Furthermore, this paper does not take into account the effect of thin film stresses developed during deposition and issues such as depoling and aging of active materials which significantly degrade the performance. Also a particular, simple, actuator design is chosen to allow a straightforward comparison of the choices of material combinations. Detailed design clearly requires more refined models for the material and actuator geometry. Nevertheless, the estimates obtained from this paper are sufficiently accurate to facilitate materials selection decisions in the preliminary design of actuators. The conclusions regarding the ranking of the material combinations are sufficiently clear cut that they will not be altered by introducing more refined models.

This paper is organized as follows. Section II discusses the optimal performance of BPE actuators. Section III focuses on the materials selection strategy for BPE actuators using performance maps. Section IV discusses the process for identifying suitable material combinations for various functional requirements. Section V summarizes the conclusions drawn from this paper.

\section{Optimal Performance of BPE Actuators}

Bimaterial actuator structures find wide applications in MEMS devices because of the simplicity of their microfabrication and their ability to provide out of plane motion. Although the mechanics of uni-material and BPE actuator structures are well documented, little attention has been given to the materials selection process. This paper focuses on identifying promising materials for BPE actuator structures applying mechanics relations developed previously [18]-[20]. Fig. 1 shows a schematic of an ideal cantilever BPE actuator. The actuator structure consists of a piezoelectric layer and an elastic substrate referred to by subscripts 1 and 2, respectively. The layers corresponding to the electrodes are neglected in the present analysis. The actuator length and width are given by $L$ and $b$, respectively. The layer thicknesses are denoted by $t_{1}$ and $t_{2}$ and $t$ is the total thickness. The Young's moduli of the bimaterials are denoted by $E_{1}$ and $E_{2}$. The ratio of the Young's moduli of the bimaterials is defined as, $\lambda=E_{1} / E_{2}$ and the ratio of the thicknesses of the bilayers, $\xi=t_{1} / t_{2}$.

Actuation in piezoelectric bilayers can be achieved either in $d_{31}$ mode or $d_{33}$ mode (using an interdigitated configuration) as shown in Fig. 2(a) and (b). The tip slope, blocked moment and maximum work per volume of the BPE actuator in $d_{31}$ mode are given as

$$
\begin{aligned}
\Theta_{\mathrm{f}} & =\frac{6 d_{31} E_{\mathrm{p}} L}{t}\left(\frac{\lambda \xi(\xi+1)^{2}}{\left(\lambda \xi^{3}+1\right)(1+\lambda \xi)+3 \lambda \xi(\xi+1)^{2}}\right) \\
M_{\mathrm{blk}} & =\frac{E_{1} b t^{2} d_{31} E_{\mathrm{p}}}{2}\left(\frac{\xi}{(1+\lambda \xi)(\xi+1)}\right) \\
W & =\frac{3 E_{1}\left(E_{\mathrm{p}} d_{31}\right)^{2}}{8\left(3(\lambda \xi+1)+\left(\frac{(1+\lambda \xi)^{2}\left(1+\lambda \xi^{3}\right)}{\lambda \xi(\xi+1)^{2}}\right)\right)\left(\frac{\xi+1}{\xi}\right)}
\end{aligned}
$$

where $E_{\mathrm{p}}=V / t_{1}$ is the electric field parallel to the polarized orientation in $\mathrm{V} / \mathrm{m}$ and $d_{31}$ is the material constant of the piezoelectric layer in $\mathrm{m} / \mathrm{V}$ or $\mathrm{C} / \mathrm{N}$. The compliance relations for BET and BPE actuator structures are the same except that the thermal strain is replaced by the piezoelectric strain. Therefore, the condition pertaining to optimal performance for a given pair of materials under a constant electric field [16], [19] is given as

$$
\lambda \xi_{\mathrm{o}}^{2}=1
$$

where $\xi_{\mathrm{o}}$ is the optimal thickness ratio corresponding to maximum performance. Substituting (4) in (1)-(3) and normalizing the geometric parameters $(L, b$, and $t)$ gives optimized critical performance metrics for a constant electric field which serves as a basis for candidate materials selection

$$
\begin{aligned}
& \Theta_{\mathrm{no}}=\frac{\Theta_{\mathrm{f}} t}{E_{\mathrm{p}} L}=\frac{6 d_{31}}{4} \\
& M_{\mathrm{no}}=\frac{M_{\mathrm{blk}}}{b t^{2} E_{\mathrm{p}}}=\frac{E_{1} d_{31}}{2\left(\frac{\xi_{\mathrm{o}}+1}{\xi_{\mathrm{o}}}\right)^{2}} \\
& W_{\mathrm{no}}=\frac{W}{E_{\mathrm{p}}^{2}}=\frac{3 E_{1}\left(d_{31}\right)^{2}}{32\left(\frac{\xi_{\mathrm{o}}+1}{\xi_{\mathrm{o}}}\right)^{2}}
\end{aligned}
$$

where $\Theta_{\text {no }}, M_{\text {no }}$, and $W_{\text {no }}$ are material parameters which correspond to optimal slope, moment and work normalized with respect to geometry and electric field.

Actuation in $d_{33}$ mode can be obtained by depositing interdigitated electrodes parallel to the polarized direction as shown in Fig. 2(b). The actuation voltage for such a configuration has to be increased by approximately five times to achieve the same performance as that obtained in $d_{31}$ mode considering the distance between the electrode fingers, $L_{\mathrm{p}} \sim 10 t_{1}$. Hence, the $d_{33}$ mode configuration is more appropriate for sensor applications than for actuator structures [21]. The present materials selection process considers only $d_{31}$ mode actuation which is commonly preferred in MEMS devices.

\section{Materials Selection Process FOR BPE ACTUATORS}

The promising active/substrate material combinations are to be identified and ranked for various performance metrics. Table I shows a list of available piezoelectric materials which might be considered for MEMS applications. The properties are 


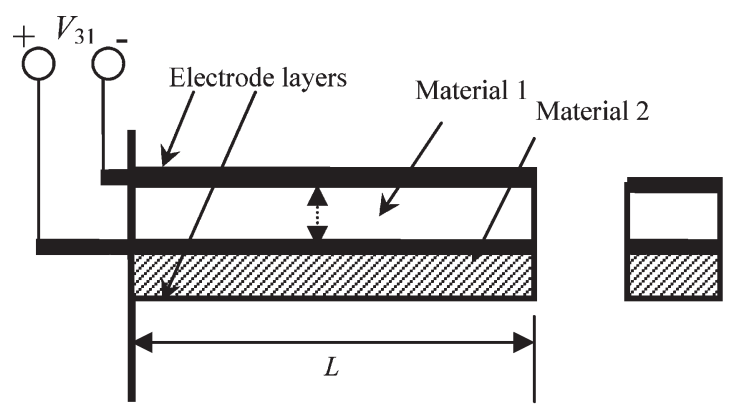

Interdigitated Electrode
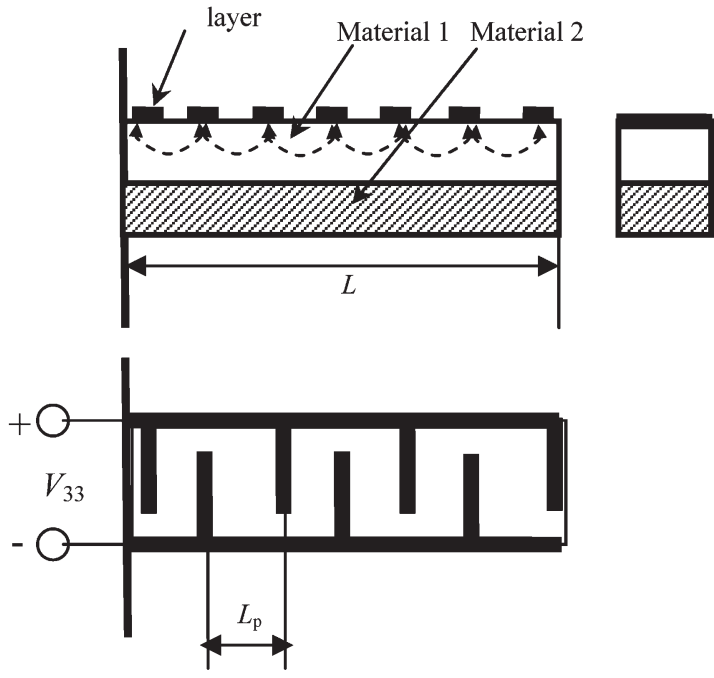

(b)

Fig. 2. Schematic of a cantilever BPE actuator. (a) Actuation by $d_{31}$ mode. (b) Actuation by $d_{33}$ mode.

obtained from the sources cited in the last column of Table I. Active materials for which sufficient data are available are only considered to allow for consideration in a practical actuator design. Both thin film and bulk properties of piezoelectric materials are presented, to indicate the variation in property values and to provide realistic estimates of what might be achieved via present and future microfabrication processes.

The materials selection strategy is based on plotting performance contours in the domain of governing active material properties for different substrate materials. Silicon is the most commonly used substrate material in MEMS devices. Therefore, the materials selection strategy is primarily illustrated using $\mathrm{Si}(E=165 \mathrm{GPa})$ substrate, but other substrates could be considered using the appropriate properties in conjunction with (5)-(7). Fig. 3(a) shows contours of equal slope $\left(\log _{10}\left(\Theta_{\text {no }}\right)\right)$, blocked moment $\left(\log _{10}\left(M_{\mathrm{no}}\right)\right)$, and work per volume $\left(\log _{10}\left(W_{\text {no }}\right)\right)$ plotted in the domain of governing properties $(d$ versus $E$ ) for a range of active materials on silicon. Similar plots are generated at the extremities of the range of substrate properties, i.e., for diamond-like carbon (DLC) $(E=700 \mathrm{GPa})$ and polymethyl methacrylate (PMMA) $(E=2.5 \mathrm{GPa})$ to provide an indication of the practical limits on performance. Fig. 3(b) and (c) shows performance contours for DLC and PMMA substrates respectively. The variation in blocked moment and work/volume are within an order of magnitude of that for $\mathrm{Si}$, while the variation in tip slope is relatively small.

Actuation frequency is another important performance metric governed partly by materials selection. BPE actuators typically operate close to mechanical resonant frequencies. The fundamental flexural frequency of a bimaterial can be estimated using Euler-Bernoulli beam theory

$$
f_{\mathrm{S}}=\frac{1}{2 \pi}\left(\frac{1.8751}{L}\right)^{2} \sqrt{\left(\frac{E_{1} t^{2}}{3 \lambda\left(\rho_{1} \xi_{\mathrm{o}}+\rho_{2}\right)\left(\xi_{\mathrm{o}}+1\right)}\right)}
$$

where $\rho_{1}$ and $\rho_{2}$ are the densities of the bimaterials. Fig. 4 shows contours of equal actuation frequency for different ma- terial combinations with respect to silicon, DLC and PMMA substrates for a length scale of $100 \mu \mathrm{m}$ and $L / t=30$. The influence of materials selection on actuation frequency is relatively small because the densities of the active materials only vary within an order of magnitude $\left(\sim 10^{3}-10^{4} \mathrm{~kg} / \mathrm{m}^{3}\right)$.

Another important target for materials selection is the actuation efficiency. Actuation efficiency is defined here as the ratio of mechanical work done/volume $(W)$ to electrical energy/volume $\left(E_{\mathrm{s}}\right)$ supplied

$$
\eta=\frac{W}{E_{\mathrm{s}}}
$$

The electrical energy supplied is assumed to be equal to the dielectric energy stored in the parallel plate capacitor, thereby ignoring electrical losses in the external circuitry (which are independent of any material selection decision). Therefore, the energy/volume supplied, $E_{\mathrm{s}}$ is given as

$$
E_{\mathrm{s}}=\frac{C V^{2}}{2 A t_{1}}=\frac{\varepsilon_{\mathrm{r}} E_{\mathrm{p}}^{2}}{2}
$$

where $\varepsilon_{\mathrm{r}}$ is the relative dielectric constant of the active material employed. Substituting (7) and (10) in (9) gives efficiency in terms of material properties

$$
\eta=\frac{3 E_{1} d_{31}^{2}}{16 \varepsilon_{\mathrm{r}}\left(\frac{\xi_{\mathrm{o}}+1}{\xi_{\mathrm{o}}}\right)^{2}}=\frac{3 K^{2}}{16\left(\frac{\xi_{\mathrm{o}}+1}{\xi_{\mathrm{o}}}\right)^{2}}
$$

where $K$ is the electromechanical coupling factor of the piezoelectric material. Fig. 5 shows the contours of equal efficiency for various piezoelectric materials plotted in the domain of governing active material properties ( $K$ versus $E$ ) for three different substrates considered. Actuation efficiency varies over three orders of magnitude $\left(\eta \sim 10^{-3}-10^{-1}\right)$ for the given range of material combinations considered.

The overall size of the integrated components for piezoelectric MEMS actuators is dependent on the off-chip voltage and 


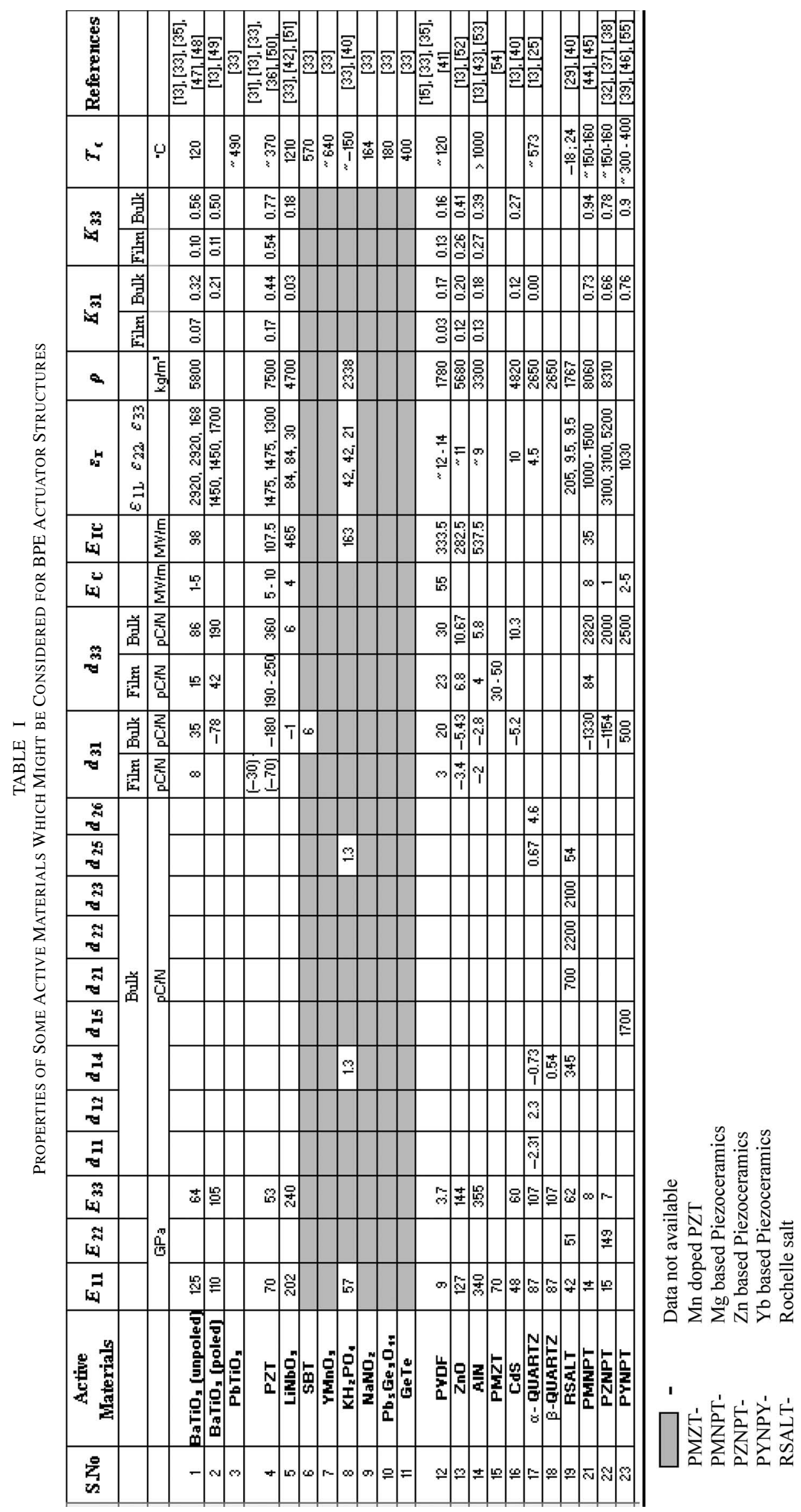




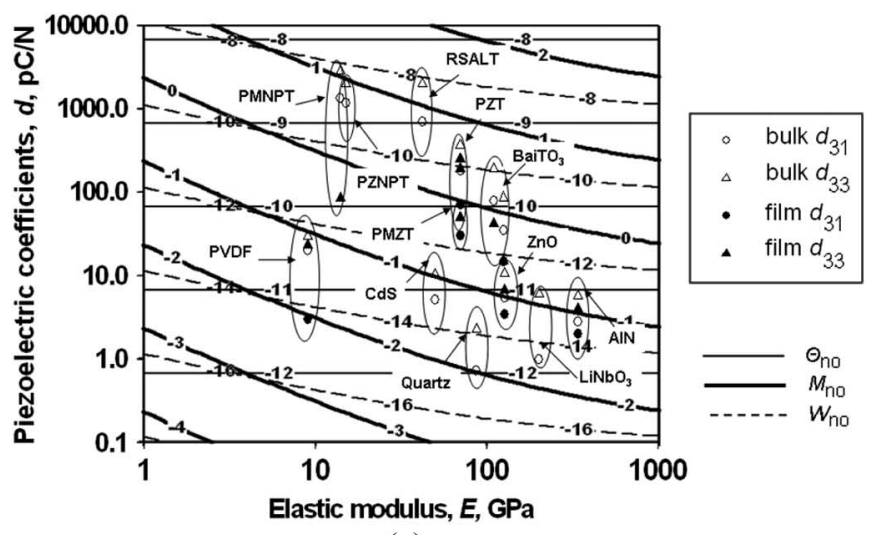

(a)

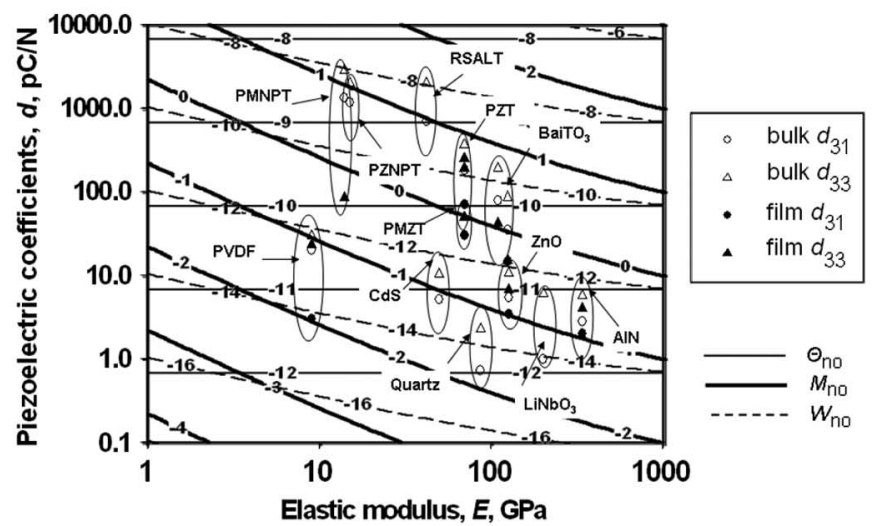

(b)

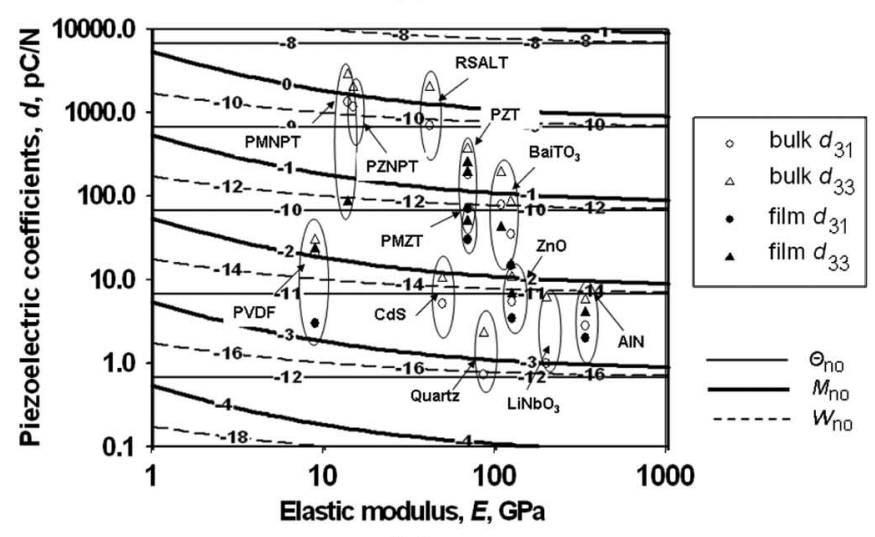

(c)

Fig. 3. Contours of equal tip slope $\left(\log _{10}\left(\Theta_{\text {no }}\right)\right)$, blocked moment $\left(\log _{10}\left(M_{\text {no }}\right)\right)$ and work per volume $\left(\log _{10}\left(W_{\text {no }}\right)\right)$ for a range of piezoelectric materials on (a) $\mathrm{Si}$, (b) DLC, and (c) PMMA.

power amplifier requirements which are prone to make the system bulky. The higher the actuation voltage, the larger the amplifier required. Hence, a comparison is made of the voltage requirements for a constant electric field with variation in active material properties over the range of substrates considered. The voltage required for actuating different BPE actuators for a constant electric field can be quantified by a parameter termed the electromechanical voltage index $\left(V_{\mathrm{I}}\right)$

$$
V_{\mathrm{I}}=\frac{V}{E_{\mathrm{p}} t}=\frac{1}{\left(1+\left(\frac{1}{\xi_{\mathrm{o}}}\right)\right)} .
$$

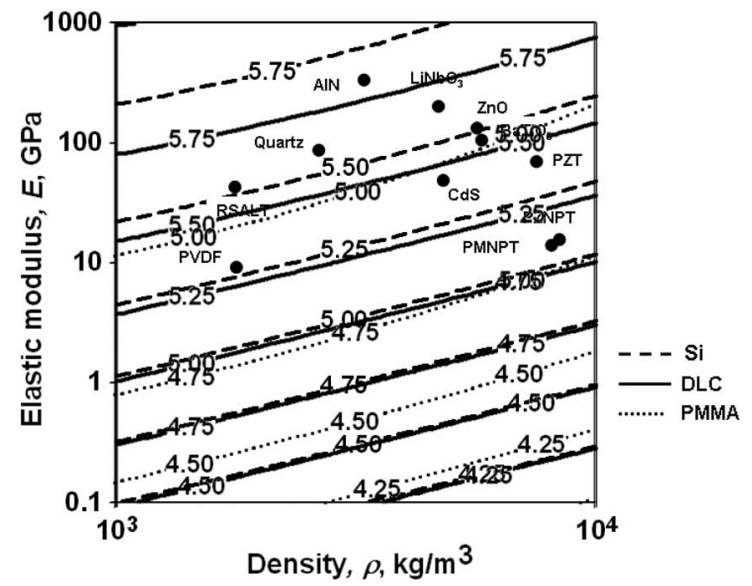

Fig. 4. Contours of equal actuation frequency $\left(\log _{10}(f)\right)$ for different piezoelectric materials on Si, DLC, and PMMA substrates.

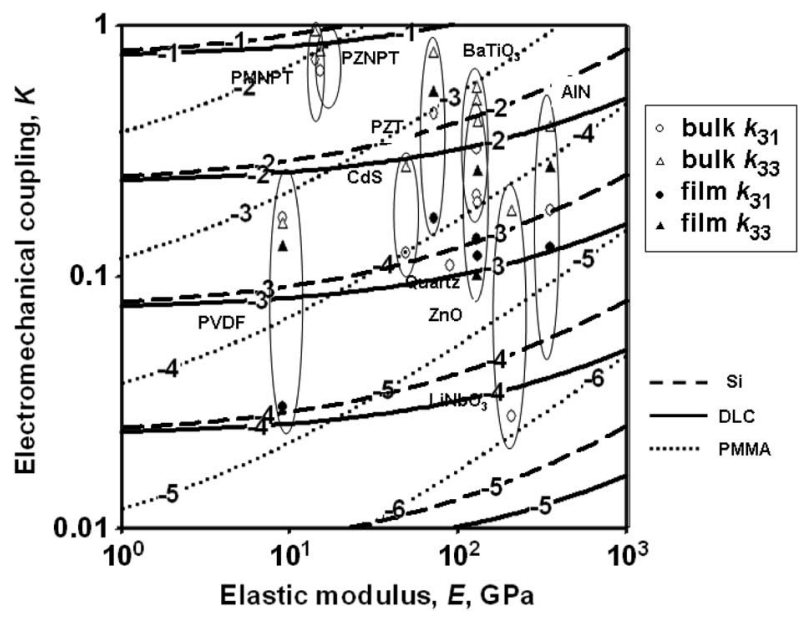

Fig. 5. Contours of electromechanical efficiency $\left(\log _{10}(\eta)\right)$ for different piezoelectric materials on $\mathrm{Si}$, DLC, and PMMA substrates.

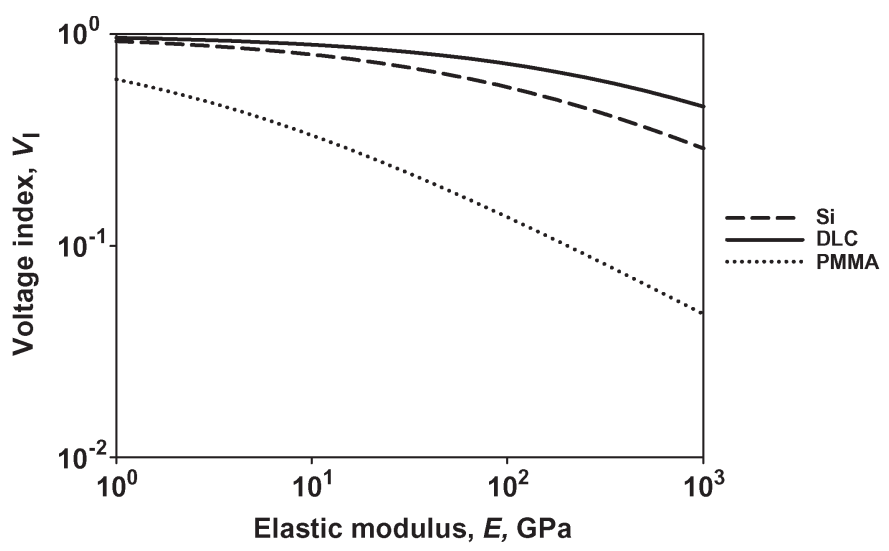

Fig. 6. Effect of different material combinations on the actuation voltage for an optimal performance under a constant electric field.

Fig. 6 shows the variation in $V_{\mathrm{I}}$ for a range of piezoelectric materials on the three different substrates considered previously. It is clear from the plot that the voltage required for a constant electric field varies by about an order of magnitude $\left(V_{\mathrm{I}} \sim\right.$ $0.1-1)$ for the range of material combinations considered. 


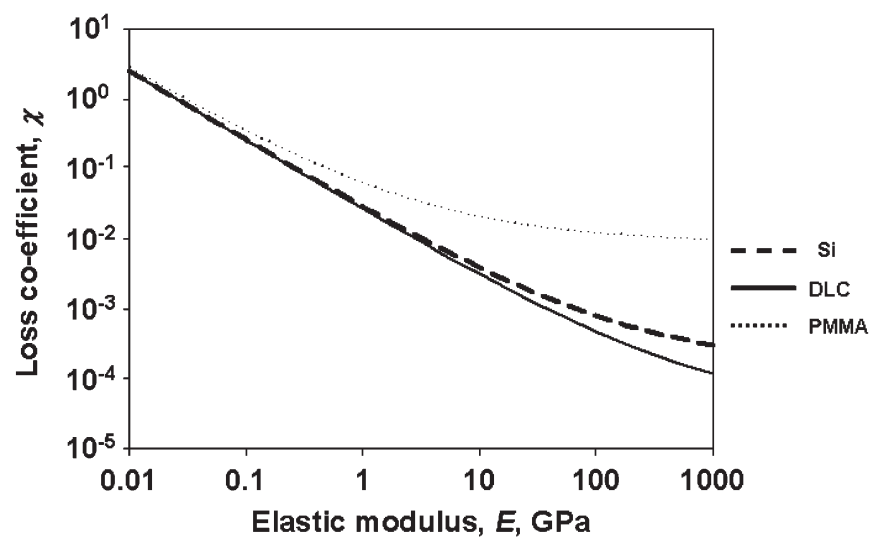

Fig. 7. Effect of material combinations on the loss coefficient (hence the $Q$-factor) of BPE actuators for a range of substrates considered.

The candidate materials selected strongly influence the quality factor (hence the loss coefficient) of the actuators. The loss coefficient, $\chi$ associated with the material damping is quantified here based on the elastic moduli of the bimaterials [22] which is a reasonably good estimate for most material combinations, except those prone to deformation by twinning (such as magnesium alloys, zinc alloys, quartz, etc.)

$$
\chi=\frac{0.1}{E_{\mathrm{eq}}}
$$

where

$$
E_{\mathrm{eq}}=\frac{E_{1} I_{1}+E_{2} I_{2}}{I}=\frac{4 E_{1}}{\left(\frac{\xi_{\mathrm{o}}+1}{\xi_{\mathrm{o}}}\right)^{2}}
$$

is the elastic modulus in gigapascal of an equivalent beam with rectangular cross section whose width is identical to that of the bilayer $(b)$ with thickness equal to the total thickness $t$. $I_{1}, I_{2}$ are the moments of inertia of each of the bilayers about the centroidal axis of the actuator structure and $I$ is the moment of inertia of the equivalent beam about its centroidal axis. Fig. 7 shows a plot of the loss coefficient of the actuator as a function of the elastic modulus of the piezoelectric material for the range of substrates considered. BPE actuator structures made of polymeric substrates have a lower $Q$-factor compared to those made of metallic or ceramic substrates. This is due to the viscous damping effects which are predominant in polymeric substrates compared to other classes of materials.

A possibly more realistic material selection strategy for an optimal dynamic response can be evolved by establishing a suitable condition for electromechanical oscillation matching the required external impedance. Fig. 8(a) shows an ideal electromechanical transduction circuit for BPE actuator structures. An input electrical signal $\left(V_{\text {in }}\right)$ applied across the terminals $\mathrm{AB}$ is transformed into an output mechanical signal $\left(F_{\text {out }}\right)$ across terminals $\mathrm{A}^{\prime} \mathrm{B}^{\prime}$ to drive the external load. The dissipation due to the leakage charges in the electrical capacitor is ignored and the input circuit therefore includes only a capacitor corresponding to the blocked electrical capacitance, $C_{\text {be }}$ parallel to the voltage source. The output circuit is the electrical analog of a lumped mechanical model for a damped vibration system. $L_{\mathrm{m}}, C_{\mathrm{m}}$, and $R_{\mathrm{m}}$ correspond to the mass, the bending stiffness and the mechanical damping constant of the bimaterial actuator structure, respectively. $\phi$ is the transduction ratio of the circuit. The parameters relevant to the electromechanical transduction are given as

$$
\begin{aligned}
C_{\mathrm{be}} & =\frac{\left(1-K^{2}\right) \varepsilon_{\mathrm{r}} L b}{t_{1}} \\
& =\left(\frac{\left(1-K^{2}\right) \varepsilon_{\mathrm{r}}\left(1+\xi_{\mathrm{o}}\right)}{\xi_{\mathrm{o}}}\right)\left(\frac{L b}{t}\right) \\
\phi & =K_{\mathrm{b}} d_{31} \\
& =\left(\frac{16 M_{\mathrm{blk}}}{3 L^{2} \Theta_{\mathrm{f}}}\right) d_{31}=\left(\frac{16 E_{1} b t^{3} \xi_{\mathrm{o}}^{2}}{9 L^{3}\left(\xi_{\mathrm{o}}+1\right)^{2}}\right) d_{31}
\end{aligned}
$$

where $K_{\mathrm{b}}$ is the bending stiffness of the bimaterial cantilever evaluated based on the average energy of the bimaterial bounded by extreme compliant conditions (fixed-free and fixed-fixed). Using the Thevenin and Norton [23] theorems, an equivalent electrical circuit of the electromechanical transduction circuit is obtained as shown in Fig. 8(b). The total impedance of the equivalent circuit, $Z_{\mathrm{T}}$ is given by

$$
Z_{\mathrm{T}}=\left(R_{\mathrm{m}}+Z_{\mathrm{L}}^{\prime}\right)+j\left(\omega L_{\mathrm{m}}-\frac{1}{\omega C_{\mathrm{m}}}-\frac{\phi^{2}}{C_{\mathrm{be}} \omega}+Z_{\mathrm{L}}^{\prime \prime}\right)
$$

where $\omega$ is the frequency of the input signal and $Z_{\mathrm{L}}^{\prime}$ and $Z_{\mathrm{L}}^{\prime \prime}$ are the real and imaginary parts of the external impedance, respectively. The output force required to overcome the external impedance is given by

$$
F_{\text {out }}=\phi V_{\text {in }} \frac{\left|Z_{\mathrm{L}}\right|}{\left|Z_{\mathrm{T}}\right|}
$$

Neglecting the mechanical damping effects in the electromechanical system, the transfer function, $G$ for the electromechanical system is given as

$$
G=\frac{F_{\text {out }}}{V_{\text {in }}}=\frac{\phi Z_{\mathrm{L}} \omega C_{\mathrm{m}} C_{\mathrm{be}}}{C_{\mathrm{be}}\left(\left(\frac{\omega}{\omega_{\mathrm{n}}}\right)^{2}-1\right)+Z_{\mathrm{L}}^{\prime \prime} \omega C_{\mathrm{m}} C_{\mathrm{be}}-\phi^{2} C_{\mathrm{m}}}
$$

where $\omega_{\mathrm{n}}$ is the undamped natural frequency of the actuator structure. It is evident from (18) that the frequency of the input signal has to be tuned for a given material combination to match the required external impedance. The external impedance depends on the nature of the system utilizing the work delivered by the actuator and this typically can be modeled as a springmass system under free or forced vibration. The physical significance of (18) is illustrated by considering a simple application such as a fiber-optic switch, where the external impedance can be modeled as a spring mass system under free vibration. For instance, a large $F_{\text {out }}$ at high $\omega_{\mathrm{n}}$ can be attained for a given input voltage $V_{\text {in }}$ only if the signal frequency $\omega$ is increased which in turn requires a large integrated power amplifier unit. A reduction in the requirements for an amplifier could be achieved if optimal materials were to be selected which would require 


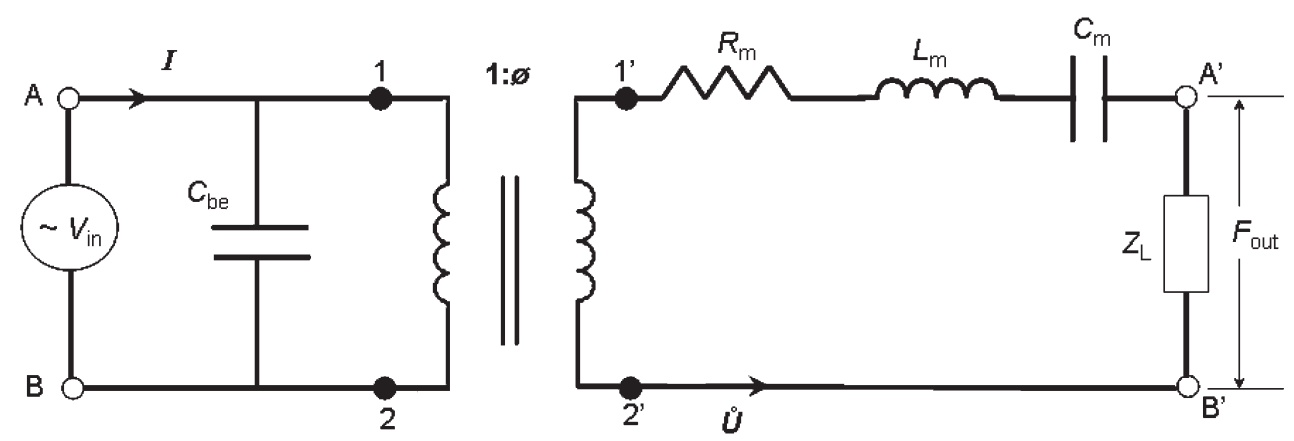

(a)

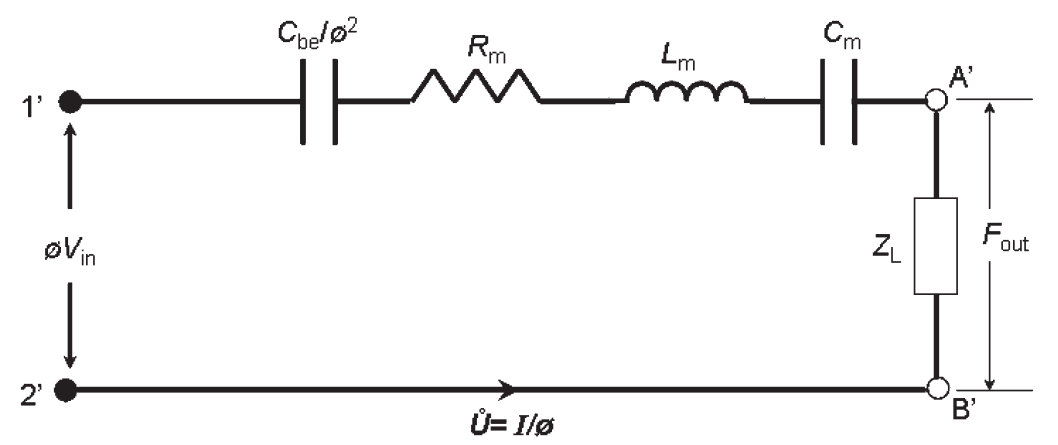

(b)

Fig. 8. (a) Ideal electromechanical transduction circuit for BPE actuators. (b) Equivalent circuit of the ideal transduction circuit obtained by applying Thevenin and Norton theorems.

evaluation of the limiting value of $G$ at $\omega=\omega_{\mathrm{n}}$. Therefore, substituting $\omega=\omega_{\mathrm{n}}$ in (18) gives

$$
G=\frac{F_{\text {out }}}{V_{\text {in }}}=\frac{Z_{\mathrm{L}}^{\prime \prime}}{\left(\frac{Z_{\mathrm{L}}^{\prime \prime}}{\phi}-\frac{\phi}{C_{\mathrm{be}} \omega_{\mathrm{n}}}\right)} .
$$

Electromechanical oscillation occurs as $G \rightarrow \infty$, i.e., the denominator of (19) should tend to zero to obtain a large $F_{\text {out }}$ for an infinitesimally small $V_{\mathrm{in}}$. Therefore, (19) reduces to

$$
Z_{\mathrm{L}}^{\prime \prime}=\frac{\phi^{2}}{C_{\mathrm{be}} \omega_{\mathrm{n}}}
$$

Equation (20) is the relevant performance metric for selecting optimal material combinations to match the external impedance. Material combinations with a large $\phi$ and a small $C_{\mathrm{be}}$ are desirable to overcome a large impedance considering the variation in $\omega_{\mathrm{n}}$ bounded within an order of magnitude. Using (14) and (15), performance indices are obtained by normalizing $\phi$ and $C_{\text {be }}$ with respect to the geometry and dielectric constant

$$
\begin{aligned}
{\left[C_{\mathrm{be}}\right]_{\mathrm{I}} } & =C_{\mathrm{be}}\left(\frac{t}{L b \varepsilon_{\mathrm{r}}}\right)=\left(\frac{\left(1-K^{2}\right)\left(1+\xi_{\mathrm{o}}\right)}{\xi_{\mathrm{o}}}\right) \\
\phi_{1} & =\frac{\phi L^{3}}{b t^{3}}=\left(\frac{16 E_{1} \xi_{\mathrm{o}}^{2}}{9\left(\xi_{\mathrm{o}}+1\right)^{2}}\right) d_{31}
\end{aligned}
$$

where $\left[C_{\mathrm{be}}\right]_{\mathrm{I}}$ and $\phi_{\mathrm{I}}$ are the capacitance index and transduction index, respectively. Figs. 9 and 10 show contours of equal $\left[C_{\mathrm{be}}\right]_{\mathrm{I}}$ and $\log _{10}\left(\phi_{\mathrm{I}}\right)$ for different piezoelectric materials on the range of substrates considered. For performance metrics described as a function of many variables, relevant nondi-

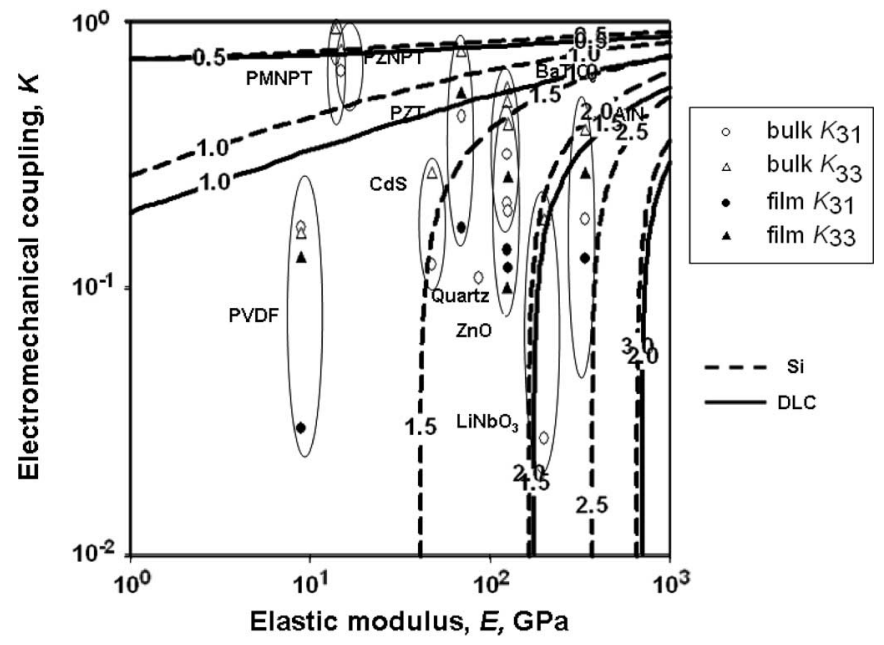

Fig. 9. Contours of blocked capacitance index $\left(\left[\mathrm{C}_{\mathrm{be}}\right]_{\mathrm{I}}\right)$ for different active materials on Si and DLC substrates.

mensional indices that dictate the materials selection can be formulated applying the Buckingham $\pi$ theorem.

\section{RESUltS AND Discussion}

Selection of suitable actuation for an application requires estimation of the performance limits achieved from different actuation methods. Piezoelectric actuation in general, is particularly promising for high force applications. It is evident from the contours shown in Fig. 3, that BPE actuators are more applicable for high force/work $\left(M_{\text {no }} \sim 10 \mathrm{~N} / \mathrm{mV}, W_{\text {no }} \sim\right.$ $\left.10^{-8} \mathrm{~N} / \mathrm{V}^{2}\right)$ applications than for large displacement applications $\left(\Theta_{\text {no }} \sim 10^{-8} \mathrm{~m} / \mathrm{V}\right)$. Although the work/volume, $W$ 


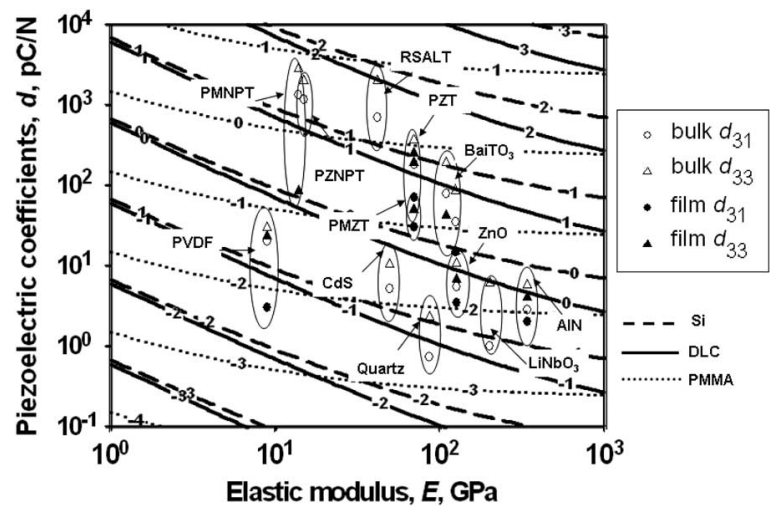

Fig. 10. Contours of equal electromechanical transduction index $\left(\log _{10}(\phi)_{\mathrm{I}}\right)$ for different active materials on the range of substrates considered.

delivered by piezoelectric actuation [2], [3], [24] is $\sim 10^{5} \mathrm{Jm}^{-3}$, the drop in the $d$ coefficient at microscales by an order of magnitude reduces $W$ by two orders of magnitude $\left(\sim 10^{3} \mathrm{Jm}^{-3}\right)$ which is evident from the results presented herein. Also from Fig. 4 it is clear that an actuation frequency of $\sim 100 \mathrm{kHz}$ can be attained for an actuator length of $100 \mu \mathrm{m}$ with $L / t=30$. Furthermore, the maximum efficiency of BPE structures is $\eta \sim 0.1$, which is an order of magnitude less than the efficiency of uni-material active structures estimated previously [2]. This reflects the internal strain energy locked within the bimaterial that is not available for performing external work.

It is apparent from Fig. 3 that ferroelectric piezoceramics dominate in performance compared to other active materials over the entire range of practical substrate materials. Quartz, traditionally used for macroactuators and sensors is not suitable for MEMS actuators due to its low piezoelectric constants [25]-[27]. Despite its very high piezoelectric constants, Rochelle salt $\left(\mathrm{NaKC}_{4} \mathrm{H}_{4} \mathrm{O}_{6} \cdot 4 \mathrm{H}_{2} \mathrm{O}\right)$ is unlikely to be practical for microactuators due to its unstable nature and low transition temperature [28]-[30]. Material combinations such as PMNPT, PZNPT, PZT and $\mathrm{BaTiO}_{3}$ on $\mathrm{Si}$, DLC or other high stiffness substrates are promising candidates for large force/work actuators with high transduction indices and reasonable efficiency $(\eta \sim 0.1)$. AlN on Si or DLC substrates is marginally superior to other material combinations for high frequency applications ( $>100 \mathrm{kHz}$ ) by virtue of the relatively high modulus of AlN. Although polymeric substrates exhibit a lower $Q$-factor than other classes of materials due to viscous damping effects, the actuation voltage required by the active materials when combined with polymers in all cases is less for a given electromechanical strain.

The present material selection strategy has certain limitations. Variations in thin film properties of materials due to processing routes, temperature and substrate effects have not been captured in the present analysis. The processing routes strongly influence the electromechanical properties of piezoceramics. For example, thin film PZT grown by sol-gel has a relatively high remnant polarization $\left(\sim 56.8 \mu \mathrm{C} / \mathrm{cm}^{2}\right)$ and a lower coercive field than films grown by a pulsed laser deposition process [31]. Furthermore, the electromechanical properties are very sensitive to stoichiometric variation of the piezoceramic constituents [32] which are not taken into account in this paper although the materials selection decision is not greatly influenced by this variation.

The operating temperature of the actuator structure significantly affects the performance. The electrical properties of active materials are sensitive to temperature variation although the change in elastic modulus is generally not significant. The active materials selected for the BPE microactuator structure should possess Curie temperatures greater than the operating temperature. The influence of Curie temperature on the materials selection process can be better understood from the data presented in Table I. The Curie temperatures for most of the active materials considered are above $200{ }^{\circ} \mathrm{C}$ except for Rochelle salt $\left(-18{ }^{\circ} \mathrm{C}\right.$ and $\left.24^{\circ} \mathrm{C}\right)$ and $\mathrm{KH}_{2} \mathrm{PO}_{4}\left(\sim-150{ }^{\circ} \mathrm{C}\right)$, which make these materials suitable only for low temperature applications. $\mathrm{LiNbO}_{3}$ and $\mathrm{AlN}$ can be considered for harsh environment applications where the operating temperature exceeds $\sim 1000{ }^{\circ} \mathrm{C}$.

The present materials selection strategy has not accounted for the variation in coercive field of the piezoelectric materials which further restricts actuator response. A desirable active material selected should possess a large coercive field while also having a high $d$ coefficient. Although the $d$ coefficients increase at the stoichiometric combinations corresponding to the morphotropic phase boundary (tetragonal-rhombohedral transition) for ferroelectric piezoceramics, this is accompanied by a drop in the coercive field [33], [34]. Furthermore, the applied electric field on the active materials reaches the maximum achievable value only if the coercive field, $E_{\mathrm{c}}$ is as high as the intrinsic coercive field, $E_{\text {ic }}$. However, this is feasible only at nanoscales considering the inverse dependence of the coercive field on the film thickness [35], [36] which thereby reduces the work done per cycle. For polymeric films, $E_{\mathrm{c}}$ approaches $E_{\mathrm{ic}}$ only at Langmuir-Blodgett scales [35] which are beyond the capability of the present microfabrication processes and results in actuators with very low absolute values of work or force delivered. For ferroelectric piezoceramic films, values of $E_{\mathrm{c}}$ up to a few megavolts per meter can be achieved at the microscale given the present capability of microfabrication. This is smaller than $E_{\mathrm{ic}}$ by an order of magnitude. Although the coercive field of piezoelectric polymers ( $E_{\mathrm{c}}$ of Polyvinylidene fluoride (PVDF) $\sim 55 \mathrm{MV} / \mathrm{m}$ ) is greater than that of the ferroelectric piezoceramics by an order of magnitude, piezoelectric polymers require a large thickness to compensate for their low elastic modulus to deliver optimal performance. This is unlikely to be acceptable for many applications.

Selection of an actuation mechanism for a given application determines the performance of MEMS devices. Table II shows the comparison of the performances of BET (Al-Si) and BPE (PZT-Si) cantilever actuator structures for sensible limits on temperature $\left(\Delta T=150^{\circ} \mathrm{C}\right)$ and electric field $\left(E_{\mathrm{p}}=\right.$ $5 \mathrm{MV} / \mathrm{m}$ ), respectively. The tip deflection, blocked moment and work/volume delivered by the BET actuators are greater than that of the BPE actuators for a fixed geometry. However, the actuation frequency of the BPE actuators is greater than that of the BET actuators by an order of magnitude. Although the force delivered by the BPE actuator structures could be improved without an appreciable drop in the actuation frequency at large scales $\left(\sim 10 \mathrm{kHz}\right.$ can be attained even for $L \sim 2 \times 10^{-3} \mathrm{~m}$ 
TABLE II

Comparison of the Performances of BPE And BET ACtuator Structures

\begin{tabular}{|l|c|c|}
\hline \multicolumn{1}{|c|}{ Actuator structure } & BET & BPE \\
\hline \multicolumn{1}{|c|}{ Bimaterials } & $\mathrm{Al}-\mathrm{Si}$ & PZT - Si \\
\hline Actuator Length, $L$ in $\mathrm{m}$ & $60 \times 10^{-6}$ & $60 \times 10^{-6}$ \\
\hline Actuator width, $b$ in $\mathrm{m}$ & $30 \times 10^{-6}$ & $30 \times 10^{-6}$ \\
\hline Actuator thickness, $t$ in $\mathrm{m}$ & $2 \times 10^{-6}$ & $2 \times 10^{-6}$ \\
\hline Temperature difference, $\Delta T$ in $\mathrm{C}$ & $\sim 150$ & 5 \\
\hline Electric field, $E_{\mathrm{p}}$ in $\mathrm{MV} / \mathrm{m}$ & - & - \\
\hline Power dissipated, $P$ in $\mathrm{W}$ & $\sim 0.1$ & $\sim 143 \times 10^{3}$ \\
\hline Dielectric energy/volume stored, $E_{\mathrm{S}}$ in $\mathrm{J} / \mathrm{m}^{3}$ & - & $\sim 5 \times 10^{-7}$ \\
\hline Tip deflection, in $\mathrm{m}$ & $\sim 5 \times 10^{-6}$ & $\sim 5 \times 10^{-10}$ \\
\hline Blocked moment, $M_{\mathrm{blk}}$ in $\mathrm{Nm}$ & $\sim 5 \times 10^{-9}$ & $\sim 293$ \\
\hline Maximum work/volume, $W$ in $\mathrm{Nm} / \mathrm{m}^{3}$ & $\sim 27410$ & $\sim 250$ \\
\hline Actuation frequency, $f$ in $\mathrm{kHz}$ & $\sim 10$ & $\sim 10^{-3}$ \\
\hline Loss coefficient, $\chi$ & - & $\sim 0.1$ \\
\hline Actuation efficiency, $\eta$ & $\sim 10^{-5}$ & \\
\hline
\end{tabular}

and $L / t=30$ ), the displacement and the work/volume which could be achieved are still less than that for the BET actuators corresponding to the assumed scale. For applications such as arrays of small flap actuators for boundary layer flow control [56], [57], it is necessary for the actuator structures to operate at high frequencies $(\sim 10 \mathrm{kHz})$ and to deliver a high work per cycle. Since the BPE actuators operate at mechanical resonance, an actuation frequency of $\sim 100 \mathrm{kHz}$ can be achieved even for a cantilever length of a few hundred micrometers; however, the work/volume per cycle is relatively small. On the other hand, BET actuators have the ability to actuate at $\sim 10 \mathrm{kHz}$ only if their length is less than $60 \mu \mathrm{m}$ [17]; however, the work/volume per cycle is larger than that of the BPE actuator. Unlike BET actuators which are characterized by high losses due to the Joule heating effect, the losses associated with BPE actuators due to charge leakage are very small. As a result, the actuation efficiency of BET actuators is less than that of BPE actuators by four orders of magnitude. However, in BPE actuators, a large amount of input electrical energy is stored within the blocked electrical capacitor without being utilized for transduction. Also, the ability of BPE actuator structures to operate at resonant frequencies can lead to energy losses due to damping effects (material, and/or fluid) which are absent in BET actuators. The BPE actuators are also prone to depoling and aging issues which degrade the performance.

Unlike BPE actuators, BET actuators require relatively simple microfabrication steps involving sputtering of $\mathrm{Al}$ on $\mathrm{SOI}$ wafers followed by etching. This involves comparably few mask patterns. BPE actuators require relatively complicated fabrication routes involving electrode deposition; spin coating of the seed layer and sol-gel deposition of the piezoceramic followed by pyrolysis and sintering [31]. Furthermore, the range of variation in the electromechanical properties (such as $d$ coefficients, coercive field, and saturated polarization) with scale is greater than that of the mechanical and thermal properties. On balance, this analysis suggests that BET actuators are (perhaps) surprisingly competitive with BPE devices, unless high frequency and/or efficiency are the primary design drivers.

\section{CONCLUSION}

An approach to the optimal materials selection for BPE actuators has been presented. Critical performance metrics are obtained by applying the mechanics of BPE actuator structures developed by earlier works. Using performance maps, optimal material combinations are identified for various functional requirements. Material combinations such as PMNPT, PZNPT, PZT and $\mathrm{BaTiO}_{3}$ on $\mathrm{Si}$ or DLC are promising for large force/work actuators with high transduction indices and efficiency $(\eta \sim 0.1)$. AIN on Si or DLC substrates performs better than other alternative candidates for high frequency applications $(\sim 600 \mathrm{kHz})$. A condition for electromechanical oscillation is obtained which serves as a useful basis for materials selection to achieve matching of the external impedance. Polymeric substrates offer higher material damping due to viscous effects compared to other classes of materials. Selection of an appropriate actuation method for a boundary layer flow control application has been illustrated by comparing the performance limits of BPE and BET actuator structures. The potential of active materials could be realized for MEMS applications if better control over the properties of thin films could be achieved during microfabrication. This can be accomplished only by enhancing our understanding of the process-property relationships from further research studies.

\section{REFERENCES}

[1] S. M. Spearing, "Materials issues in microelectromechanical systems (MEMS)," Acta Mater., vol. 48, no. 1, pp. 179-196, Jan. 2000.

[2] J. E. Huber, N. A. Fleck, and M. F. Ashby, "The selection of mechanical actuators based on performance indices," Proc. R. Soc. Lond. A, Math. Phys. Sci., vol. 453, no. 1965, pp. 2185-2205, Oct. 1997.

[3] D. J. Bell, T. J. Lu, N. A. Fleck, and S. M. Spearing, "MEMS actuators and sensors: Observation on their performance and selection for purpose," J. Micromech. Microeng., vol. 15, no. 7, pp. S153-S164, Jul. 2005.

[4] Y. Takahashi and M. Suzuki, "Piezoelectric inkjet printer using laminated piezoelectric actuator," U.S. Patent 5402 159, Mar. 28, 1995.

[5] R. Maeda, J. J. Tsaur, S. H. Lee, and M. Ichiki, "Piezoelectric microactuator devices," J. Electroceramics, vol. 12, no. 1/2, pp. 89-100, Jan. 2004.

[6] J. Friend, A. Umeshima, T. Ishii, K. Nakamura, and S. Ueha, "A piezoelectric linear actuator formed from a multitude of bimorphs," Sens. Actuators, A, Phys., vol. 109, no. 3, pp. 242-251, Jan. 2004. 
[7] P. J. Rayner, S. A. Wilson, R. W. Whatmore, and M. Cain, "An automated performance testing system for piezoelectric micromotors," in Proc. IET Semin. MEMS Actuators Sens., London, U.K., 2006, pp. 233-236.

[8] S. Dong, S. P. Lim, K. H. lee, J. Zhang, L. C. Lim, and K. Uchino, "Piezoelectric ultrasonic micromotor with $1.5 \mathrm{~mm}$ diameter," IEEE Trans. Ultrason., Ferroelectr., Freq. Control, vol. 50, no. 4, pp. 361-367, Apr. 2003

[9] S. Priya, H. Kim, S. Ural, and K. Uchino, "Errata-High power universal piezoelectric transformer," IEEE Trans. Ultrason., Ferroelectr., Freq. Control, vol. 53, no. 4, pp. 810-816, Apr. 2006.

[10] X. Yang, Z. Zhou, H. Cho, and X. Luo, "Study on PZT-actuated diaphragm pump for air supply for micro fuel cells," Sens. Actuators, A, Phys., vol. 130/131, pp. 531-536, Aug. 2006.

[11] D. C. Roberts, H. Li, L. Steyn, O. Yaglioglu, S. M. Spearing, M. A. Schmidt, and N. W. Hagood, "A piezoelectric microvalve for compact high-frequency, high-differential pressure hydraulic micropumping systems," J. Microelectromech. Syst., vol. 12, no. 1, pp. 81-92, Feb. 2003.

[12] S. R. Platt, S. Farritor, and H. Haider, "On low-frequency electric power generation with PZT ceramics," IEEE/ASME Trans. Mechatronics, vol. 10, no. 2, pp. 240-252, Apr. 2005.

[13] H. Jaffe and D. A. Berlincourt, "Piezoelectric transducer materials," Proc. IEEE, vol. 53, no. 10, pp. 1372-1386, Oct. 1965.

[14] R. F. S Hearmon, "The elastic constants of piezoelectric crystals," $B r . J$. Appl. Phys., vol. 3, no. 4, pp. 120-124, Apr. 1952.

[15] J. G. Gualtieri, J. A. Kosinski, and A. Ballato, "Piezoelectric materials for acoustic wave applications," IEEE Trans. Ultrason., Ferroelectr, Freq. Control, vol. 41, no. 1, pp. 53-59, Jan. 1994.

[16] S. Prasanna and S. M. Spearing, "Materials selection and design of microelectrothermal bimaterial actuators," J. Microelectromech. Syst., vol. 16, no. 2, pp. 248-259, Apr. 2007.

[17] P. Srinivasan and S. M. Spearing, "Effect of heat transfer on materials selection for bimaterial electrothermal actuators," J. Microelectromech. Syst., to be published.

[18] D. L. DeVoe and A. P. Pisano, "Modeling and optimal design of piezoelectric cantilever microactuators," J. Microelectromech. Syst., vol. 6, no. 3, pp. 266-270, Sep. 1997.

[19] Q. M. Wang and L. E. Cross, "Performance analysis of piezoelectric cantilever bending actuators," Ferroelectrics, vol. 215, pp. 187-213, 1998.

[20] Q. M. Wang, X. H. Du, B. Xu, and L. E. Cross, "Electromechanical coupling and output efficiency of piezoelectric bending actuators," IEEE Trans. Ultrason., Ferroelectr., Freq. Control, vol. 46, no. 3, pp. 638-646, May 1999.

[21] Y. Jeon, R. Sood, L. Steyn, and S. G. Kim, "Energy harvesting MEMS devices based on $\mathrm{d}_{33}$ mode piezoelectric $\mathrm{Pb}(\mathrm{Zr}, \mathrm{Ti}) \mathrm{O}_{3}$ thin film cantilever," in Proc. CIRP Semin. Micro Nano Technol., Copenhagen, Denmark, 2003.

[22] D. Cebon and M. F. Ashby, "Materials selection for precision instruments," Meas. Sci. Technol., vol. 5, no. 3, pp. 296-306, 1994.

[23] J. D. Irwin and R. M. Nelms, Basic Engineering Circuit Analysis, 8th ed. Hoboken, NJ: Wiley, pp. 173-175.

[24] E. T. Carlen and C. H. Mastrangelo, "Electrothermally activated paraffin microactuators," J. Microelectromech. Syst., vol. 11, no. 3, pp. 165-174, Jun. 2002.

[25] R. Bechmann, "Elastic and piezoelectric constants of alpha-quartz," Phys. Rev., vol. 110, no. 5, pp. 1060-1061, Jun. 1958

[26] A. Mullen, "Temperature variation of the piezoelectric constant of quartz," J. Appl. Phys., vol. 40, no. 4, pp. 1693-1696, Mar. 1969.

[27] S. Noge and T. Uno, "Measurement of piezoelectric and elastic stiffness constants of $\beta$ phase quartz at high temperature region," in Proc. IEEE Ultrason. Symp., 1998, pp. 585-597.

[28] J. Valasek, "Piezo-electric and allied phenomena in Rochelle salt," Phys. Rev., vol. 17, no. 4, pp. 475-481, Apr. 1921.

[29] A. O. dos Santos et al., "Rochelle salt piezoelectric coefficients obtained by X-ray multiple diffraction," J. Phys.: Condensed Matter, vol. 13, no. 46, pp. 10497-10 505, Nov. 2001

[30] W. G. Cady, "The longitudinal piezoelectric effect in Rochelle salt crystals," in Proc. Phys. Soc., 1937, vol. 49, pp. 646-653.

[31] S. K. Pandey, A. R. James, C. Prakash, T. C. Goel, and K. Zimik, "Electrical properties of PZT thin films grown by sol-gel and PLD using a seed layer," Mater. Sci. Eng. B, vol. 112, no. 1, pp. 96-100, Sep. 2004.

[32] R. Zhang, B. Jiang, W. Jiang, and W. Cao, "Complete set of properties of $0.92 \mathrm{~Pb}\left(\mathrm{Zn}_{1 / 3} \mathrm{Nb}_{2 / 3}\right) \mathrm{O}_{3}-0.08 \mathrm{PbTiO}_{3}$ single crystal with engineered domains," Mater. Lett., vol. 57, no. 7, pp. 1305-1308, Jan. 2003

[33] R. Waser, Nanoelectronics and Information Technology, 2nd ed. Hoboken, NJ: Wiley, 2005, pp. 61-78.

[34] M. Iwata and Y. Ishibashi, "Theory of morphotropic phase boundary in solid solution systems of perovskite-type oxide ferroelectrics: Engineered domain configurations," Jpn. J. Appl. Phys., vol. 39, no. 9A, pp. 51565163, Sep. 2000

[35] V. M. Fridkin and S. Ducharme, "General features of the intrinsic ferroelectric coercive field," Phys. Solid State, vol. 43, no. 7, pp. 1320-1324, Jul. 2001.

[36] N. A. Pertsev, J. R. Contreras, V. G. Kukhar, B. Hermanns, H. Kohlstedt, and R. Waser, "Coercive field of ultrathin $\mathrm{Pb}\left(\mathrm{Zr}_{0.52} \mathrm{Ti}_{0.48}\right) \mathrm{O}_{3}$ epitaxial films," Appl. Phys. Lett., vol. 83, no. 16, pp. 3356-3358, Oct. 2003.

[37] M. Sitti, D. Campolo, J. Yan, and R. S. Fearing, "Development of PZT and PZN-PT based unimorph actuators for micromechanical flapping mechanisms," in Proc. IEEE Int. Conf. Robot. Autom., Seoul, Korea, 2001, pp. 3839-3846.

[38] J. Yin and W. Cao, "Effective macroscopic symmetries and materials properties of multidomain $0.955 \mathrm{~Pb}\left(\mathrm{Zn}_{1 / 3} \mathrm{Nb}_{2 / 3}\right) \mathrm{O}_{3}-0.045 \mathrm{PbTiO}_{3}$ single crystals," J. Appl. Phys., vol. 92, no. 1, pp. 444-448, Jul. 2002.

[39] S. Zhang, L. Lebrun, S. Rhee, C. A. Randall, and T. R. Shrout, "Dielectric and piezoelectric properties as a function of temperature for $\mathrm{PB}\left(\mathrm{Yb}_{1 / 2} \mathrm{Nb}_{1 / 2}\right) \mathrm{O}_{3}-\mathrm{PbTiO}_{3}$ single crystals," in Proc. 13th IEEE Int. Symp. Appl. Ferroelectr., 2002, pp. 455-458.

[40] C. S. Brown, R. C. Kell, R. Taylor, and L. S. Thomas, "Piezoelectric materials, a review of progress," IRE Trans. Compon. Parts, vol. 9, no. 4, pp. 193-211, Dec. 1962.

[41] H. Wang, Q. M. Zhang, L. E. Cross, and A. O. Sykes, "Piezoelectric, dielectric and elastic properties of poly (vinylidene fluoride/ trifluoroethylene)," J. Appl. Phys., vol. 74, no. 5, pp. 3394-3398, Sep. 1993.

[42] A. W. Warner, M. Onone, and G. A. Coquin, "Determination of elastic and piezoelectric constants for crystals in class ( $3 \mathrm{~m})$," J. Acoust. Soc. Amer. vol. 42, no. 6, pp. 1223-1231, Dec. 1967.

[43] I. L. Guy, S. Muensit, and E. M. Goldys, "Extensional piezoelectric coefficients of gallium nitride and aluminum nitride," Appl. Phys. Lett., vol. 75, no. 26, pp. 4133-4135, Dec. 1999.

[44] R. Zhang, B. Jiang, and W. Cao, "Elastic, piezoelectric and dielectric properties of multidomain $0.67 \mathrm{~Pb}\left(\mathrm{Mg}_{1 / 3} \mathrm{Nb}_{2 / 3}\right) \mathrm{O}_{3}-0.33 \mathrm{PbTiO}_{3}$ single crystals," J. Appl. Phys., vol. 90, no. 7, pp. 3471-3475, Oct. 2001.

[45] H. Maiwa and N. Ichinose, "Electrical and electromechanical properties of $\mathrm{PB}\left(\mathrm{Mg}_{1 / 3} \mathrm{Nb}_{2 / 3}\right) \mathrm{O} 3(50 \%)-\mathrm{PbTiO}_{3}(50 \%)$ thin films prepared by chemical solution deposition," Jpn. J. Appl. Phys., vol. 42, no. 2A, pp. 850-854, 2006.

[46] S. J. Zhang, S. Rhee, C. A. Randall, and T. R. Shrout, "Dielectric and piezoelectric properties of high curie temperature single crystals in the $\mathrm{PB}\left(\mathrm{Yb}_{1 / 2} \mathrm{Nb}_{1 / 2}\right) \mathrm{O}_{3}-\mathrm{PbTiO}_{3}$ solid solution series," Jpn. J. Appl. Phys., vol. 41, no. 2A, pp. 722-726, 2002.

[47] I. D. Kim, Y. Avrahami, and H. L. Tuller, "Study of orientation effect on nanoscale polarization in $\mathrm{BaTiO}_{3}$ thin films using piezoresponse force microscopy," Appl. Phys. Lett., vol. 86, no. 19, pp. 192 907-1-192 907-3, May 2005.

[48] H. B. Sharma and A. Mansingh, "Sol-gel processed barium titanate ceramics and thin films," J. Mater. Sci., vol. 33, no. 17, pp. 4455-4459, Sep. 1998.

[49] M. Sayer, "Piezoelectric thin film devices," in Proc. IEEE Ultrason. Symp., 1991, pp. 595-603.

[50] C. Ayela, L. Nicu, C. Soyer, E. Cattan, and C. Bergaud, "Determination of the $\mathrm{d}_{31}$ piezoelectric coefficient of $\mathrm{PbZr}_{\mathrm{X}} \mathrm{Ti}_{1-\mathrm{X}} \mathrm{O} 3$ thin films using multilayer buckled membranes," J. Appl. Phys., vol. 100, no. 5, pp. 054908 1-054 908-9, Sep. 2006.

[51] S. Kim, V. Gopalan, and A. Gruverman, "Coercive fields in ferroelectrics: A case study in lithium niobate and lithium tantalate," Appl. Phys. Lett., vol. 80 , no. 15 , pp. $2740-2742$, Apr. 2002

[52] K. Tanaka, R. Kubo, K. Ohwada, A. Umeda, K. Ueda, and T. Usuda, "Measurement of piezoelectric constant of $\mathrm{ZnO}$ film on Si microstructure," Jpn. J. Appl. Phys., vol. 34, no. 9B, pp. 5230-5232, Sep. 1995.

[53] P. E. J. Sebastain, "Monitoring of refractory wall recession using high temperature impact-echo instrumentation," U.S. Dept. Energy, Washington, DC, Rep. UDR-TR-2004-00018, Contract DE_FG36$02 \mathrm{GO} 12051$

[54] J. E. A. Southin, S. A. Wilson, D. Schmitt, and R. W. Whatmore, " $\mathrm{e}_{31, \mathrm{f}}$ determination for PZT films using conventional ' $\mathrm{d}_{33}$ ' meter," J. Phys. $D$, Appl. Phys., vol. 34, no. 10, pp. 1456-1460, May 2001.

[55] Q. F. Zhou, Q. Q. Zhang, and S. T. McKinstry, "Structure and piezoelectric properties of sol-gel-derived (001)-oriented $\mathrm{Pb}\left[\mathrm{Yb}_{1 / 2} \mathrm{Nb}_{1 / 2}\right]_{3}-\mathrm{PbTiO}_{3}$ thin films," J. Appl. Phys., vol. 94, no. 5, pp. 3397-3402, Sep. 2003.

[56] S. A. Jacobson and W. C. Reynolds, "Active control of streamwise vortices and streaks in boundary layers," J. Fluid Mech., vol. 360, no. 1, pp. 179211, Apr. 1998.

[57] J. Kim, "Control of turbulent boundary layers," Phys. Fluids, vol. 15, no. 5, pp. 1093-1105, 2003. 


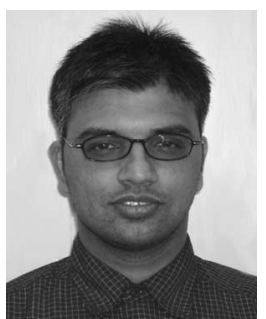

Prasanna Srinivasan received the M.S. degree in mechanical engineering from the Indian Institute of Technology_Madras, Chennai, India, in 2000.

He was a Design Engineer for GE aircraft engines global engineering unit in Bangalore, India, from 2000 to 2003, where he was a member of the structural design team in development engine programs. He is currently a Postgraduate Research Scholar in the Materials Research Group within the School of Engineering Sciences, University of Southampton, Southampton, U.K. His research interests include design and analysis of structures at micro/nanoscales, materials and structures for the design of MEMS devices, mechanics of carbon nanotubes structures, solid mechanics, heat transfer, and fluid flow analysis. He is currently involved in research that focuses on optimal materials selection for improving the performance of MEMS devices.

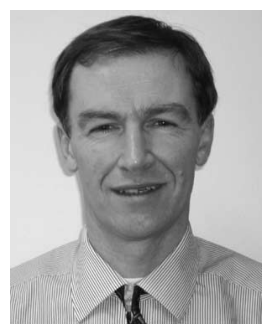

S. Mark Spearing received the Ph.D. degree from the Department of Engineering, Cambridge University, Cambridge, U.K., in 1990.

Since 2004, he has been a Professor of Engineering Materials in the School of Engineering Sciences, University of Southampton, Southampton, U.K. Prior to this, he spent ten years as a Professor of Aeronautics and Astronautics at Massachusetts Institute of Technology (MIT), Cambridge. His technical interests include materials characterizations and structural analysis and design of MEMS, development of wafer-bonding technologies, microelectronic and MEMS packaging, and advanced composites. From 1995 to 2005, he was responsible for materials, structural design, and packaging tasks of the MIT MicroEngine, MicroRobot, Micro-Chemical Power, and MicroHydraulic Transducer projects as well as conducting cross-cutting underpinning technology development.

Dr. Spearing is an Associate Editor of the Journal of Microelectromechanical Systems and a member of the American Society of Mechanical Engineers. In 2004, he received a Royal Society Wolfson Research Merit Award. 